\title{
Detecting Glaucoma by Various Image Processing Techniques
}

\author{
Sukrita N $S^{1}$, Dr. Sindhu $\mathbf{R}^{2}$ \\ PG Student, Communication Engineering, NSS College of Engineering, Palakkad, India ${ }^{1}$ \\ Professor, Electronics and Communication Engineering, NSS College of Engineering, Palakkad, India ${ }^{2}$
}

\begin{abstract}
Glaucoma is a very serious case of eye disease which may lead to permanent and irreversible blindness if left untreated. The retinal image analysis involves usage of various structural and texture features of retinal images. Various image processing techniques are used for the detection of glaucoma. Some of its key elements to detect glaucoma includes image registration, fusion, segmentation, feature extraction, enhancement, pattern matching, image classification, analysis and various statistical measurements. The main idea behind this paper is to describe various image processing techniques for the detection of glaucoma. As years go by, number of medical assistants are reducing whereas the demand for health care is increasing. The methods mentioned in this review has its own advantages and disadvantages. Success rate of each method is mentioned. These methods will be helpful for one to choose an optimum method in future. Once glaucoma is detected and diagnosed, probability of total permanent blindness in the patients having glaucomatous eye can be avoided.
\end{abstract}

Keywords: Glaucoma, Segmentation, Enhancement, Feature Extraction, Fundus Images, Retinal Images

\section{INTRODUCTION}

Glaucoma is one of the ocular diseases which leads to irreversible blindness. According to a survey about $2.3 \%$ of the total population got affected by glaucoma and it is predicted to affect around 11.1 million people (around $2.86 \%$ ) by 2020 [1]. Among various retinal diseases $13 \%$ of cases are being affected by glaucoma. Glaucoma is a very serious case of eye disease as it shows no symptoms in earlier stages. There will be no pain or vision changes in its early stages. If it remains untreated, the patient may start losing his vision which gradually leads to blindness. Glaucoma is non reversible but can be controlled by detecting the disease in earlier stages. In the case of advanced stages of glaucoma, patient usually shows thinness of the cornea, high eye pressure and abnormal optic nerve anatomy. Glaucoma is mainly caused due to the increased intraocular pressure in the eye. Human eye produces a small amount of fluid called aqueous fluid or aqueous humour to maintain a healthy vision. It provides nutrition for various tissues. In normal eye amount fluid produced and amount of fluid thrown out of the eye are same.
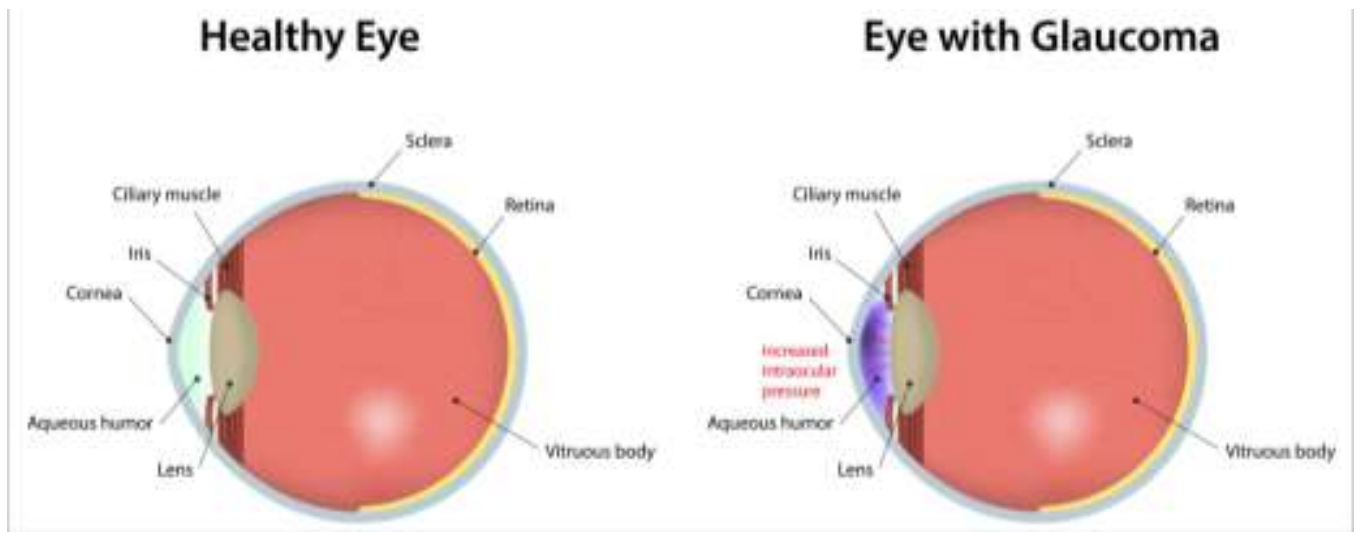

Fig 1: Normal and Glaucoma affected eye[3]

But when drainage system is blocked, eye produces an excess of fluid which creates an imbalance in the fluid flow. This leads to an increase in intraocular pressure[2]. Poor blood regulation of the optic nerve also causes pressure variation in the optic nerve. Due to this increased pressure in the optic nerve glaucoma results leading to nerve damage and vision loss. So early detection and diagnostics of glaucoma is important to prevent serious vision loss. Various parameters that may result in glaucoma includes increased intraocular pressure, abnormality in the visual field and changes in the retinal structure. Various structural changes that occur due to glaucoma includes variation in the optic 


\title{
International Advanced Research Journal in Science, Engineering and Technology
}

\author{
Vol. 6, Issue 4, April 2019
}

nerve head. Retina consists of millions of nerve fibres which groups together to form the optic nerves. Various structural and textural information about a retina is mentioned in Fig. 1. Signs and symptoms of glaucoma is also mentioned below.

\section{A. Some anatomical structures of retina}

1. Optic Disc: Optic nerves that enter into the round region of the retina is termed as optic disc.

2. Fovea: The depression in the retina that contains only cones and provides accurate vision.

3. Macula: Area in the middle of the retina that allows seeing object with great detail.

4. Anterior Chamber: It lies between the cornea and the lens. It is filled with a fluid known as aqueous humor. It is also known as anterior cavity.

5. Aqueous humor: It is a transparent watery fluid that circulates in the anterior chamber. Aqueous humor Provides nutrients and oxygen to the inner eye and exerts fluid pressure which helps in maintaining the shape of the eye.

\section{B. Signs and symptoms of Glaucoma}

1. Peripheral vision is gradually lost[4]. This nearly always affects both eyes.

2. In advanced stages, the patient has tunnel vision.

3. Blurred vision.

4. Red eyes.

5. Sudden unexpected vision problems, especially when lighting is poor.

\section{GLAUCOMA DETECTION}

Various number of clinical methods are available to detect glaucoma pathology, but the population growth and raising levels of obesity causes direct examination, a limiting factor for the ophthalmologists. So an automatic recognition system for the characteristics of pathological cases would provide a great benefit

\section{A. Process of detecting Glaucoma}

Initially, retinal images are captured using digital devices before starting the glaucoma detection process. Then preprocessing is performed for equalizing and reshaping the irregularities on the images. Feature extraction process is performed after pre-processing stage to reduce the dimensions effectively for describing the large data set precisely. Pixel intensity values, textures, Histogram model etc are the methods used in feature extraction. Image Classification is performed which analysis the numerical properties of an image and organizes the data. Depending on the results obtained, the set of data is used to check whether the retinal image is normal or glaucomatous.

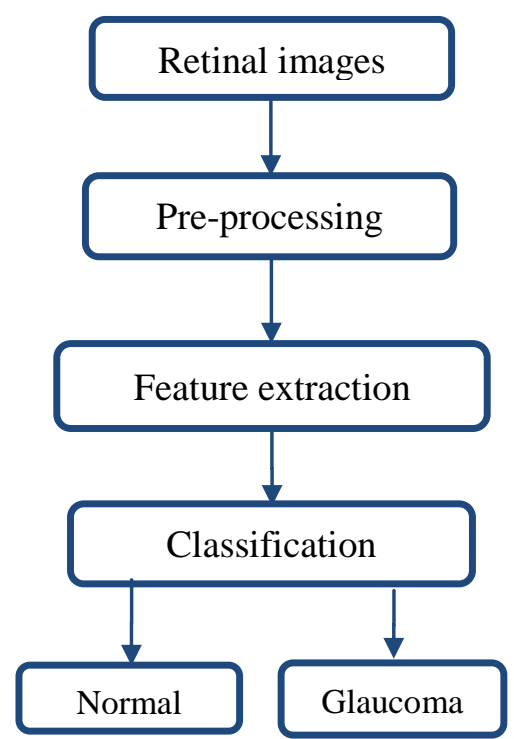

Fig 2: General process of detecting glaucoma[5]

\section{B. Techniques and algorithms for the detection of glaucoma}

\section{Using wavelet features of segmented optic disc from fundus image}

It is an automatic image processing based method for glaucoma detection from the digital fundus image. Here wavelet feature extraction has been followed by optimized genetic feature selection combined with several learning algorithms 


\title{
International Advanced Research Journal in Science, Engineering and Technology
}

\author{
Vol. 6, Issue 4, April 2019
}

and various parameter settings. It indicates that the wavelet features of the segmented optic disc image are clinically more significant in comparison to features of the whole or sub fundus image in the detection of glaucoma from fundus image. Here optic disc is localized and segmented using bit plane analysis. After optic disc segmentation and blood vessel removal wavelet features re extracted. Prominent feature selection and glaucoma classification is performed. Accuracy of glaucoma identification achieved in this work is $94.7 \%$ [6]. The noisy blood vessels were removed from the segmented optic disc using this method and the features were extracted from this de-noised image. May be use of other feature selection methods and different classification method leads to more improved accuracy and efficiency.

\section{Detection using CDR(cup to disc ratio)}

Retina has ganglion cells scattered all over it. They have fibers that converges on the optic nerve head and layers of fibers get thicker just at their nerve head which is piled up and dive into the opening. The nerve head is a disc filled with fibers, and the left over space in the middle of nerve head is called cup. Comparing the size of cup to the size of whole disc is Cup to Disc Ratio (CDR) [7\& 8]. Due to increase in intra ocular pressure, the CDR increases as the cup size increases. The CDR is considered to be less than 0.5 for normal optic disc but for abnormal optic disc, it exceeds 0.5.The increase in cup size also affects the Neuro-retinal Rim (NRR). NRR is the area located between the edge of the optic disc and the optic cup[9]. A novel sparse dissimilarity-constrained coding (SDC) approach is used for glaucoma detection. It considers both the dissimilarity constraint and the sparsity constraint from a set of reference discs with known CDR. Subsequently, the reconstruction coefficients from the SDC are used to compute the CDR for the testing disc. This method has been tested for glaucoma screening. The SDC formulation combines the pixel-wised difference in data term with the dissimilarity term. It is shown that by including the disc dissimilarity constraint this method significantly improves the CDR computation. The limitation is that the method may not capture local cup deformation.

\section{Super pixel classification technique for glaucoma screening.}

This method [10] involves developing glaucoma screening technique using super pixel classification on optic disc and optic cup segmentation. In this method, each optic disc image is first segmented into super pixels. Then from each of the super pixel, mean intensities, center surround locations and features of the location has been extracted in order to classify whether it is a cup or non-cup. In optic disc segmentation, histogram has been used to differentiate each super pixel as disc or non-disc. For optic cup segmentation, location information is also included along with the histograms and center statistics. This method showed an over-lapping error of $9.5 \%$ and $24 \%$ in optic disc and optic cup.

\section{Using PCA and mathematical morphology}

The goal is to facilitate the early detection of certain pathologies. The method used for the extraction of the optic disc here is mainly based on mathematical morphology along with principal component analysis (PCA)[11]. The input of the segmentation method is obtained through PCA. The purpose of using PCA is to achieve the grey-scale image that better represents the original RGB image. Here PCA is applied on the RGB image to get a grey level image where the optic disc, vessels and different structures of retina is differentiated more clearly. Several operations based on mathematical morphology are implemented with the aim of locating the OD. It is used to make the early detection of diseases related to the fundus easier. But if the lesion is as big as the optic disc and brighter, the method may fail.

\section{Using Texture and Higher Order Spectra Features}

It is a novel method for glaucoma detection which uses a combination of texture and higher order spectra (HOS) features from digital fundus images[12]. HOS provides both amplitude and phase information of a given signal. Support vector machine, sequential minimal optimization, naïve Bayesian, and random- forest classifiers are used to perform supervised classification. It is found that the random-forest classifier, combined with z-score normalization and featureselection methods, performed the best among the four classifiers with a classification accuracy of more than $91 \%$. The classification accuracy can be further improved by increasing the number of diverse training images, choosing better features and better classifiers and using controlled environmental lighting conditions during image acquisition. Using more diverse digital fundus images from normal and glaucoma subjects can further enhance the percentage of correct diagnosis.

\section{Using Two Dimensional DWT(Discrete wavelet transform).}

It is a system for glaucoma analysis using texture features extracted from retinal images. The texture features are extracted from the retinal images using two dimensional DWT[13]. Five different wavelet filters are used in this method. Image features are of two types. They are structural features and texture features. Structural features are features related to structure such as shape and size of various parts in image. Texture features depends on pixel values, which includes average or different energy values. Here in this method features are extracted using two dimensional DWT. Discrete wavelet transforms gives information about both special and frequency information of a signal. Texture feature extraction can be done efficiently by wavelet transforms. From the extracted features a range is defined for each feature of normal images. The images are said to be with abnormality if the energy feature value is out of the defined range. This features extraction method using DWT gives a classification accuracy of $91.66 \%$. Various methods can be 


\section{International Advanced Research Journal in Science, Engineering and Technology}

Vol. 6, Issue 4, April 2019

combined with this method to improve efficiency and accuracy.

\section{Detection of glaucoma without segmentation}

It is a classification system which does not depend on any segmentation methods. Image features are provided so to detect glaucoma and based on that features image is classified as normal or abnormal[14]. Three classifiers are used here which are Naïve Bayes, K nearest neighbor and support vector machine. It is standard pattern recognition method. Using this method features are analyzed and used to capture the structure of glaucoma. This system shows an accuracy rate of $86 \%$.

\section{Datasets used \\ 1. RIGA(Retinal fundus Image for Glaucoma Analysis)[15] \\ 2. HRF (High Resolution Fundus)[16] \\ 3. Drishti_GS1[17][18]}

\section{CONCLUSION}

The visual impairment caused by glaucoma is not reversible. This ocular disorder damages the optic nerve which is a major nerve for vision. There are no such noticeable symptoms that helps for the detection of glaucoma in the early stages[19]. Manual based glaucoma detection is costly, time consuming and prone to measurement error. So automatic detection techniques of glaucoma have introduced. In this paper various methods have been discussed for the detection of glaucomatous eye in its early stages. As it is very important to detect glaucoma and take required diagnostics methods to avoid permanent blindness. Fundus images of the eye is used in various image processing techniques for its detection. The above methods used for detecting glaucoma are compared and the performance measurement of each method have been discussed. These methods may be helpful for the people living in the less developed areas where the number of ophthalmologist is less. Various new method with more accuracy and less complexity can be introduced in future for the early detection of glaucoma.

\section{REFERENCES}

[1]. Chethan Kumar N.S, Dr. Somashekar K, "Image Processing Techniques for Automatic Detection of Glaucoma- A Study", International Journal of Latest Technology in Engineering, Management \& Applied Science (IJLTEMAS), Volume VI, Issue VII, July 2017.

[2]. M. Caroline Viola Stella Mary, Elijah Blessing Rajsingh And Ganesh R. Naik," Retinal Fundus Image Analysis for Diagnosis of Glaucoma: A Comprehensive Survey", IEEE Access, vol. 4, July 2016.

[3]. Available: https://www.magrudereye.com/glaucoma-orlando/

[4]. M. D. Abramoff, M. K. Garvin, and M. Sonka, "Retinal imaging and image analysis," IEEE Trans. Med. Imag., vol. 3, pp. 169-208, 2010.

[5]. Tehmina Khalil, Samina Khalid and Adeel M, Syed „Review of Machine Learning Techniques for Glaucoma Detection and Prediction” Science and Information Conference, August 2014, London, UK.

[6]. Anushikha Singh, Malay Kishore Dutta, M. ParthaSarathi, Vaclav Uher, Radim Burget, Image processing based automatic diagnosis of glaucoma using wavelet features of segmented optic disc from fundus image, Computer methods and programs in biomedicine 124, Elseveir (2016), 108-120.

[7]. Jun Cheng Fengshou Yin, Damon Wing Kee Wong., "Sparse Dissimilarity- Constrained Coding for Glaucoma Screening” IEEE Transactions on Biomedical Engineering, Vol. 62, No. 5, May 2015.

[8]. Hafsah Ahmad, Abubakar Yamin, Aqsa Shakeel, Syed Omer Gillani, Umer Ansari „,Detection of Glaucoma Using Retinal Fundus Images ${ }^{\text {9e }}$ 9781- 4799-5132-1/14/\$31.00 @2014 IEEE.

[9]. http://www.glaucoma.org/glaucoma/types-of glaucoma.php.

[10]. Jun cheng, Jiang liu, Y. Xu, F. Yin, D. W. K. Wong, N. M. Tan, D. Tao, C. Y. Cheng, T. Aung, and T. Y. Wong, "Super pixel classification based optic disc and optic cup segmentation for glaucoma screening”, IEEE transactions on medical imaging, vol. 32 , No. 6, June 2013.

[11]. Sandra Morales, Valery Naranjo, Jesús Angulo, and Mariano Alcaniz, "Automatic Detection of Optic Disc Based on PCA and Mathematical Morphology", IEEE Transactions on medical imaging, Vol. 32, No. 4, APRIL 2013.

[12]. U. Rajendra Acharya, Sumeet Dua, Xian Du, "Automated Diagnosis of Glaucoma Using Texture and Higher Order Spectra Features", IEEE Transactions On Information Technology In Biomedicine, May 2011, pp 449-455.

[13]. Kiran S M , Dr. D N Chandrappa, “Automatic Detection of Glaucoma Using 2-D DWT", International Research Journal of Engineering and Technology (IRJET), Volume: 03 ,Issue: 06, June-2016.

[14]. Rudiger Brock, Jorg Meier, Georg Michelson, Laszlo G Nyul and Joachim Hornegger, “ Classifying Glaucoma With Image- Based Features From Fundus Photograph”, DAGM 2007, LNCS 4713, pp. 355-364, 2007. Springer-Verlag Berlin Heidelberg 2007.

[15]. Ahmed Almazroa, Sami Alodhayb, Essameldin Osman, Eslam Ramadan, Mohammed Hummadi, Mohammed Dlaim, Muhannad Alkatee, Kaamran Raahemifar, Vasudevan Lakshminarayanan, "Retinal fundus images for glaucoma analysis: the RIGA dataset", Proc. SPIE 10579, Medical Imaging 2018: Imaging Informatics for Healthcare, Research, and Applications, 105790B (6 March 2018); doi: 10.1117/12.2293584.

[16]. Budai, Attila Bock, Rudiger; Maier, Andreas; Hornegger, Joachim; Michelson, Georg. "Robust Vessel Segmentation in Fundus Images". International Journal of Biomedical Imaging, vol. 2013.

[17]. Sivaswamy J, S. R. Krishnadas, Arunava Chakravarty, Gopal Dutt Joshi, Ujjwal and Tabish Abbas Syed , “A Comprehensive Retinal Image Dataset for the Assessment of Glaucoma from the Optic Nerve Head Analysis”, JSM Biomedical Imaging Data Papers, 2(1):1004, 2015.

[18]. Sivaswamy J, Krishnadas K. R, Joshi G. D, Jain Madhulika, Ujjwal and Syed Abbas T., "Drishti-GS: Retinal Image Dataset for Optic Nerve Head(ONH) Segmentation.”, IEEE ISBI, Beijing.

[19]. Chethan Kumar N.S, Dr. Somashekar K, "Image Processing Techniques for Automatic Detection of Glaucoma- A Study", International Journal of Latest Technology in Engineering, Management \& Applied Science (IJLTEMAS), Volume VI, Issue VII, July 2017. 